\title{
Application of 3D Human Motion in the Sports based on Computer Aided Analysis
}

\author{
Xuesi Huang*
}

Gannan Normal University, GanZhou, JiangXi, China

\begin{abstract}
D human motion tracking is in recent years the field of machine vision is a very important research direction, it has a wide range of applications, such as human-computer interaction, intelligent animation, video surveillance and other. Currently about three-dimensional human motion tracking research mostly based multicast video, monocular video due to the depth information of the lack of the 3D human motion tracking is more difficult, and most of them monocular $3 \mathrm{D}$ video motion tracking only consider is parallel to the lens without occlusion of the movement. In view of the above problems using particle filtering method based on the Monte Carlo method, and combined with a priori knowledge of the human motion for monocular 3D human motion tracking is realized. Experiments show that under the complex background with rotating movements up tracking 100 frames or so, on the long time series of 3D human motion tracking and certain difficulty, need to add more complex motion prediction model and the human body mechanical constraints to guide track.
\end{abstract}

Keywords: 3D human animation, human model, human motion constraint, inverse kinematics, monocular video, motion tracking.

\section{INTRODUCTION}

Along with the rapid development of computer hardware, especially the continuous upgrading of the graphics processor, the computational performance of the ordinary PC with ladder type of ascension, the graphics processing ability has been an unprecedented rapid increase, in recent ten years, computer graphics technology has been a leap forward development. Now, the animators can already be configured in the high-end graphics hardware of PC, with Maya, $3 \mathrm{~d}$ max software tools, to create realistic virtual digital characters, special environment and they can imagine any virtual world [1]. In the production of virtual digital characters, the character has a very important realistic motion. Usually case, make realistic character animation need professional animators to carefully design and continuous adjustment, which will cost a lot of manpower and material resources [2].

Motion capture technique is an effective method to obtain the human motion data has been more and more widely applied to human animation and game production. With all kinds of motion capture system, professional personnel has been a lot of human motion data, after some post processing and editing work, and has accumulated abundant human motion data [3]. However, the vast majority of motion capture systems require special and expensive hardware, some requirements of key parts of the body to wear signs, and is connected with the computer of the sensor, the movement of people additional the many obstacles and restrictions, and often only in a fixed environment application [4]. At present, we are not able to such motion capture technology application in such as sports, dance performances and other outdoor or more complex, the natural environment of human motion data acquisition. Motion capture should be readily available, cheap and non intrusive, any actor in any set of motion should can be captured [5].

This paper studied the extraction and 3D animation of 3D human pose in monocular videos spanning two difficulties. Based on the 2D joint locations have been obtained under the assumption that, in a scaled orthographic projection model approximate camera imaging process, according to the continuity of motion obtained the only variable factor and the relative 3D coordinates of the joints is calculated according to the proportion of human skeleton constraints. Finally, the inverse kinematics of each joint of the rotating Euler angle can be obtained. Virtual tree type joint topology is established in accordance with the standard, according to the joint Euler angle drive virtual human achieve continuous animation effect. In the study, construct the prototype system, and get good results.

\section{RELATED METHOD AND THEORY}

\subsection{Research difficulties of 3D human motion tracking}

Driven by the needs of a wide range of applications, many researchers are carried out the research of video human motion analysis [6]. However, even from the point of view of the latest research results, from the video directly to automatically obtain human motion data is still faced with many problems, one of the major difficulties from lost in the information inherent in the human body and the movement of high complexity and camera imaging process [7]. In addition, video body frequently occluded or self occlusion, used to obtain video camera and its temporal and spatial resolu- 


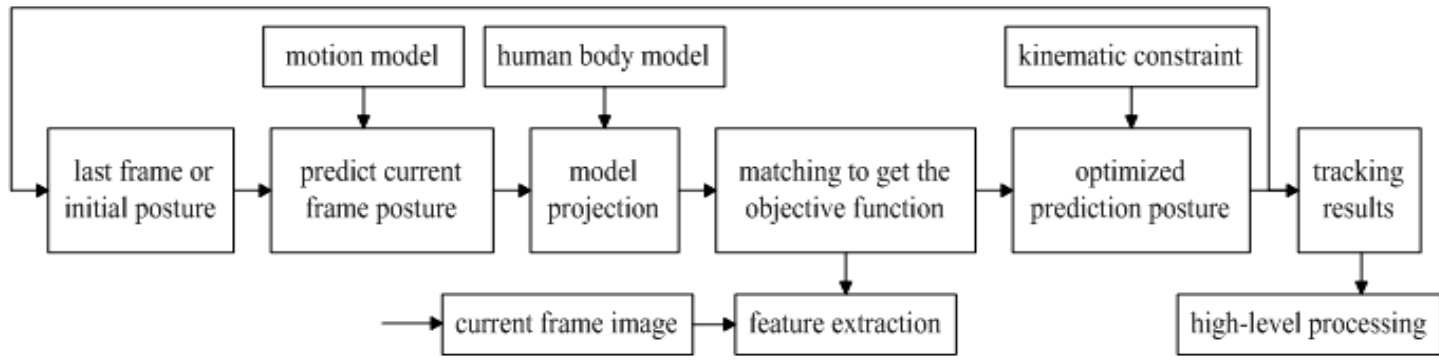

Fig. (1). Top - down model-based human motion tracking processing framework.

tion is limited, and so on objective factors also makes from the video sequence tracking and acquisition of human motion is still a difficult technical challenge. Using top-down method to capture the analysis, synthesis, tracking is in predicting matching loop corrections implemented. The general processing framework was shown in Fig. (1).

\subsection{Related Algorithm}

\section{(1) Rotational axis representation}

Rotational axis representation(Axis-angle or Exponential map), the rotation is represented as a $1 \times 3$ vector of $\mathrm{N}$.

Rotation vector $\mathrm{N}$ is expressed around the rotating axis a retatipg $\left(a_{x}, a_{z}\right)^{T \theta}$, where $\theta=\|\mathrm{a}\|$ is the $\mathrm{N}$ mode, $\mathrm{N}=\theta$.a. The corresponding rotation matrix $\mathrm{RN}(3 \times 3)$ can be calculated by Rodrigues formula (1).

$R_{a}(\theta)=e^{\bar{a}}=I_{\theta}+\sin (\theta) \hat{a}+(1-\cos (\theta)) a^{-1}, \quad \hat{a}=\left(\begin{array}{ccc}0 & -a_{z} & a_{y} \\ a_{z} & 0 & -a_{x} \\ -a_{y} & a_{x} & 0\end{array}\right)(1)$

(2) Euler angle representation

The Euler angles (phi, kappa, omega) is defined as a turn around the $\mathrm{X}, \mathrm{Y}, \mathrm{Z}$ three axes of rotation (M.shao.C.L.Nikias. et al, 1993; T.H Liu et al, 2004). The Euler rotation method a rotating object represented respectively around the $\mathrm{X}, \mathrm{y}$, and $\mathrm{Z}$ transform three axis rotation angle three. The rotation matrix is $\operatorname{Rz}(\omega) \operatorname{Ry}(\varphi) \operatorname{Rx}(k)$. The formula is the formula (2).

(3) Quaternion representation

The rotation is represented as a quaternion element vector $e=\left(e_{v}, e_{s}\right)$. Where $e_{v}=\left(e_{x}, e_{y}, e_{z}\right)$ is a vector of quaternion, $e_{s}$ is the scalar part. According to the operation of the plural, definition quaternion mode, product and conjugate are $\|e\|=\sqrt{e_{v}^{T} e_{v}+e_{s}^{2}}, \quad$ ef $=\left(e_{s} f_{v}+f_{s} e_{v}+e_{v} \cdot f_{v}, e_{s} f_{s}-e_{v}^{T} f_{v}\right)$, $\bar{e}=\left(-e_{v}, e_{s}\right)$. This is defined as quaternion $e=(n \cos (\theta / 2), \sin (\theta / 2))$ of the point $\mathrm{p}$ rotational $\theta$ angle around the axis $\mathrm{n}$. The rotation of the results can be calculated with the multiplication of quaternion.
$\left(p^{T}, 0\right)=e f \bar{e}=\left(e_{s}-e_{v}^{T} e_{v}\right) p+2\left(e_{s}\left(e_{v} \times p\right)\right.$

$\left.+\left(p^{T} e_{v}\right) e_{v}\right)$, in it $f=(p, 0)$

\subsection{The Improvement of Basic Principle of the Method of Moving Target Detection}

Covariation concept is put forward by Miller in 1998, it is similar to the covariance of Gaussian random process [8]. Random variables $X$ and $Y$ is defined as:

$[X, Y]_{\alpha}=\int_{s} x y^{<\alpha-1>} \mu(d s)$

where $\mathrm{S}$ denotes the unit circle, $\langle\bullet\rangle$ denotes the operation $z^{<\alpha>}=|z|^{\alpha} \operatorname{sign}(z)$, the covariation coefficient of $X$ and $Y$ is defined as

$\lambda_{X Y}=\frac{[X, Y]_{\alpha}}{[Y, Y]_{\alpha}}$

If the dispersion coefficient of $Y$ is $\gamma_{y}$, the covariation coefficient can be written as

$$
\begin{aligned}
& {[X, Y]_{\alpha}=\frac{E\left(X Y^{<p-1>}\right)}{E\left(|Y|^{p}\right)} \gamma_{y}} \\
& \lambda_{X Y}=\frac{E\left(X Y^{<p-1>}\right)}{E\left(|Y|^{p}\right)}
\end{aligned}
$$

According to the definition of covariation coefficient, the covariation coefficient of a real observation sequence $X(n)(n=0,1, \ldots N)$ can be defined as:

$\lambda(m)=\frac{E\left(X(n) X(n+m)^{<p-1>}\right)}{E\left(|X(n+m)|^{p}\right)}, 1 \leq p<\alpha \leq 2$

\section{EXPERIMENTAL RESULTS}

\subsection{The Human Body Geometric Modeling}

Virtual human modeling, refers to the virtual human in computer generated space geometric representation, its purpose is virtual human in the virtual environment occupied by

$$
R-R_{z} R_{y} R_{x}\left(\begin{array}{ccc}
\cos (k) \cos (\phi) & \cos (k) \sin (\phi) \sin (\omega)-\sin (k) \cos (\omega) & \cos (k) \sin (\phi) \cos (\omega)+\sin (k) \sin (\omega) \\
\sin (k) \cos (\phi) & \sin (k) \sin (\phi) \sin (\omega)+\cos (k) \cos (\omega) & \sin (k) \sin (\phi) \cos (\omega)-\cos (k) \sin (\omega) \\
-\sin (\phi) & \cos (\phi) \sin (\omega) & \cos (\phi) \cos (\omega)
\end{array}\right)
$$




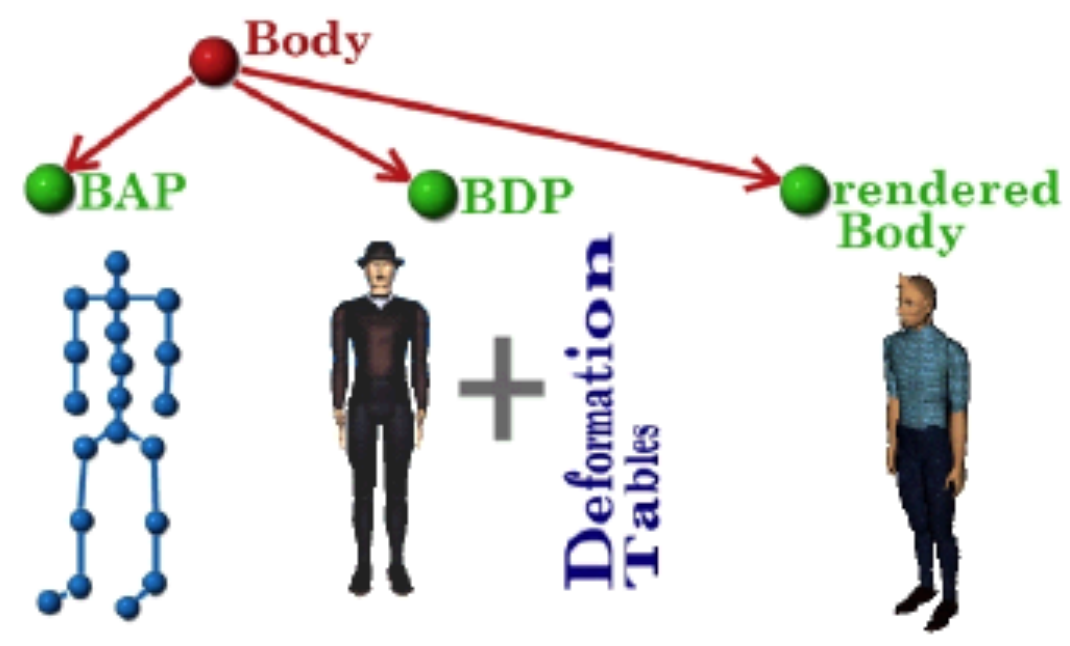

Fig. (2). Expression of virtual human in MPEG4 map.

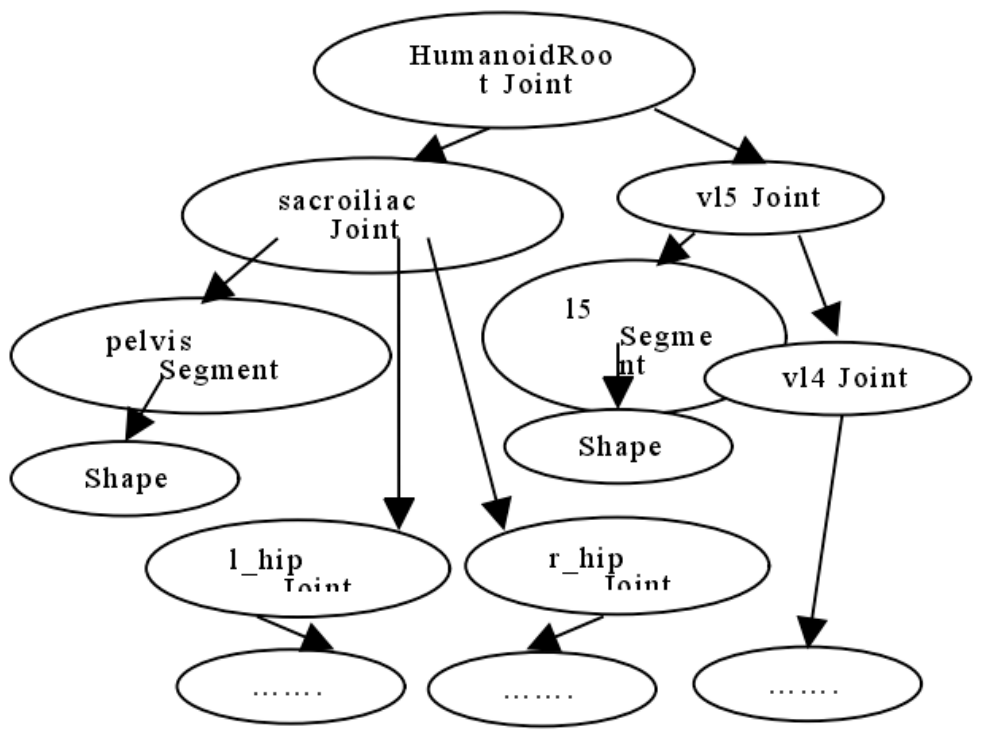

Fig. (3). Virtual tree structure.

the geometry of the space representation in computer generated space to create virtual computing graphical model, and the geometric said must meet the three-dimensional virtual humanoid in appearance and behavior characteristics of realistic requirements. In order to make the virtual people vivid and easy to drive, now uses a hierarchical method to represent virtual human [9-12].

MPEG body is composed by a group of nodes (nodes), the top node BodyNode at least comprises two child nodes: representation of human motion parameters (BAP) and defining the body model parameters (BDP), as shown in Fig. (2).

Virtual human data structure can be used in multi tree to represent, and a virtual human model can be simplified was shown in Fig. (3), figure shape node for body geometry data storage unit.

\subsection{Experiment and Analysis}

Experiment in accordance with the $\mathrm{H}$-anim standard to establish virtual tree type joint topology structure, virtual human according to the joint rotation Euler angle of anima- tion. Fig. (4) is a hip-hop movement after the reconstruction of human animation result, Fig. (5) is a reconstruction of artistic gymnastics human animation results. As shown from the first frame, every 10 frame reconstruction results. In the figure, (a) is the actual video frames and (b) is the reconstructed 3D human animation of the official figure, (c) is a $3 \mathrm{D}$ reconstruction of human body animation rotated 45 degrees of view.

\section{CONCLUSION}

Three-dimensional sports in recent years has become a hot research problem in the field of computer graphics, virtual reality, intelligent human computer interface, etc.. The research results has been widely applied to computer games, special effects in movies, training simulation system, medical aided analysis and auxiliary equipment design many fields. With the development of the visual media and humancomputer interaction technology advances by leaps and bounds, we believe that 3D human animation as a natural and harmonious interaction will have a more broad application prospects, and bring greater influence to people's life. 


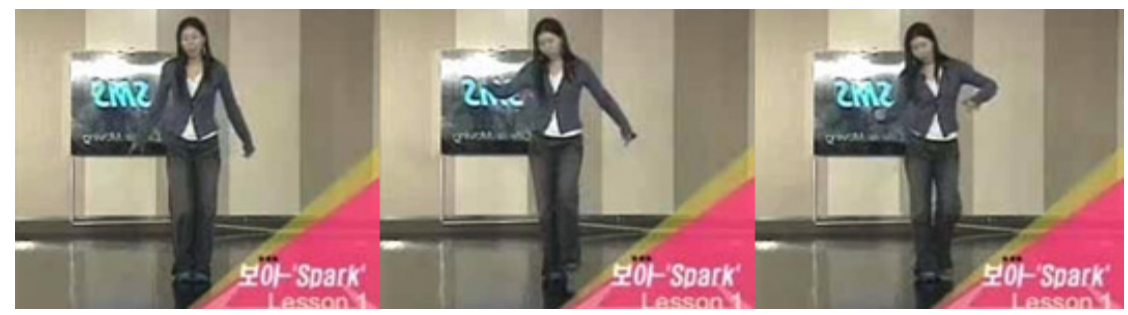

(a)
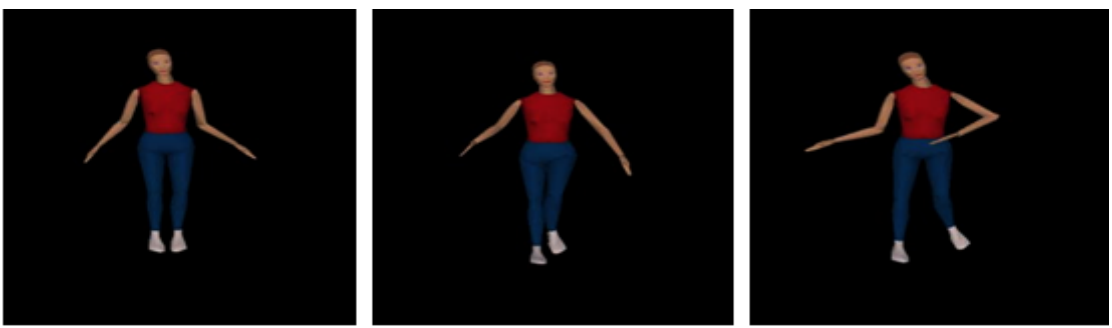

(b)
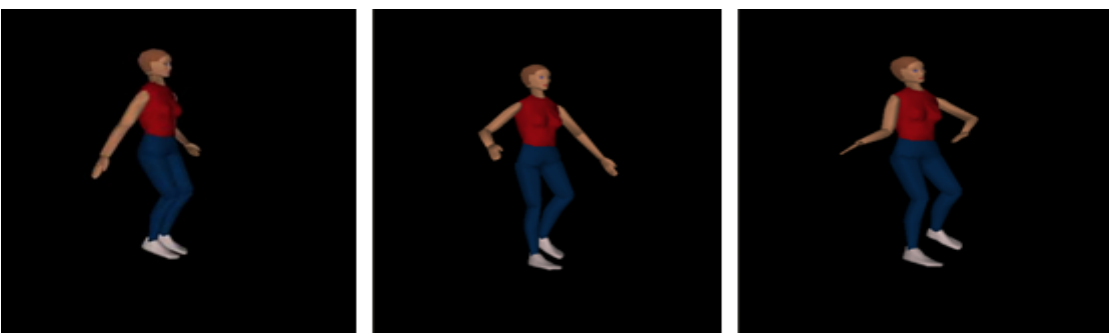

(c)

Fig. (4). Hip-hop movement after the reconstruction of human animation result.

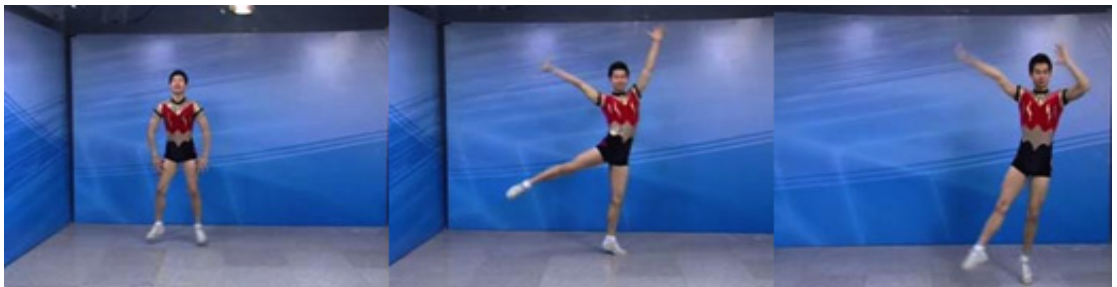

(a)

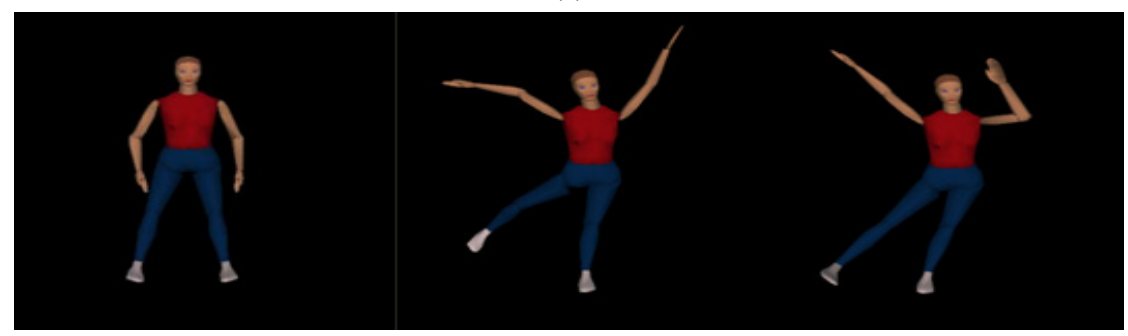

(b)

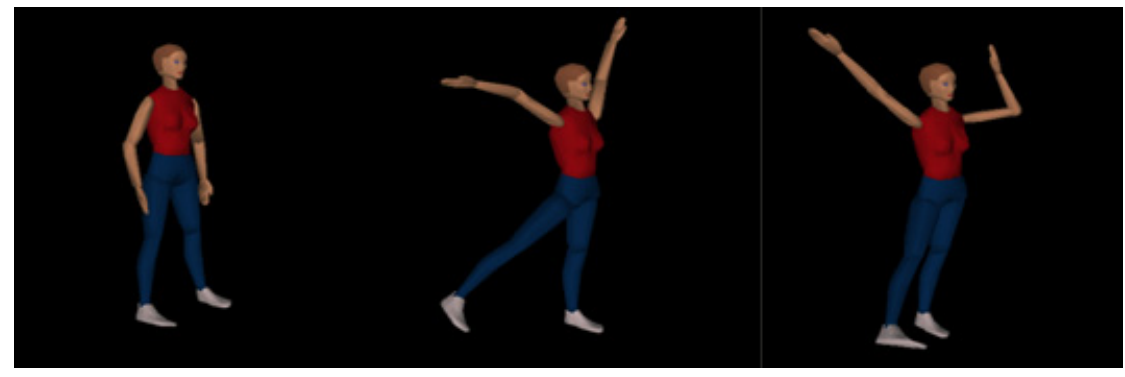

(c)

Fig. (5). Reconstruction of artistic gymnastics human animation results 


\section{CONFLICT OF INTEREST}

The author confirms that this article content has no conflict of interest.

\section{ACKNOWLEDGEMENTS}

Declared none.

\section{REFERENCES}

[1] K. Fukunaga, and L. Hostetler, "The Estimation of the Gradient of a Density Function with Applications in Pattern Recognition," IEEE Trans on Information Theory, vol. 21, no. 1, pp. 32 - 40, 1975.

[2] Y. Cheng, and S. Mean, "Mode Seeking and Clustering," IEEE Trans on Pattern Analysis and Machine Intelligence, vol. 17, no. 8pp. 790 - 799, 1995.

[3] D. Comaniciu, P. Meer, and S. Mean, "A Robust Approach toward Feature Space Analysis," IEEE Trans on Pattern Analysis and Machine Intelligence, vol. 24, no. 5, pp. 603-619, 2002.

[4] L. Gorelick, M. Blank, E. Shechtman, M. Irani, and R. Basri, "Actions as Space-Time Shapes," IEEE Trans on Pattern Analysis and Machine Intelligence, vol. 29, no. 12, pp. 2247 - 2253, 2000.

[5] P. Dollar, V. Rabaud, G. Cottrell, and S. Belongie, "Behavior Recognition via Sparse Spatio-Temporal Features," In: Proc. of the 2nd Joint IEEE International Workshop on Visual Surveillance and Performance Evaluation of Tracking and Survillance, Beijing, China, pp. $65-72,2005$.

[6] I. Laptev, and T. Lindeberg, "Space-Time Interest Points," In: Proc of the 9th IEEE International Conference on Computer Vision, Nice, France, pp. $432-439,2003$

[7] M. Jachan, G. Matz, and F. Hlawatsch, "Least-squares and Maximum-likelihood TFAR Parameter estimation for nonstarionary processes," IEEE ICASSP-2006, vol. 3, pp. 492-495, 2006.

[8] M. Jachan, G. Matz, and F. Hlawatsch, "Vector Time-Frequency AR Models for Nonstationary Multivariate Random Processes," Ieee Transactions On Signal Processing, vol. 57, no. 12, pp. 46464658, 2009.

[9] X. Ma, and C. L. Nikias, "Parameter estimation and blind channel identification in impulsive signal environments," IEEE Transactions on Signal Processing, vol. 43, no. 12, pp. 2884 - 2897, 1995.

[10] M. Shao, C. L. Nikias, "Singnal processing with fractional lower order moments instable processed and their applications," Proceedings Of The IEEE, vol. 81, no. 7, pp. 986-1010 1993, 1995.

[11] T.H. Liu, and J. M Mendel, "A Subspace-Based Direction Finding Algorithm Using Fractional Lower Order Statistics," Ieee Transactions On Signal Processing, vol. 49, no. 8, pp. 1605-1613, 2001.

[12] E. N. Kuruoglu, "Signal Processing in Stable Noise Environments: A Least lp-Norm Approach," Britain Signal Processing and Communication Laboratory, Department of Engineering, University of Cambridge, 1998.

(C) Xuesi Huang; Licensee Bentham Open.

This is an open access article licensed under the terms of the (https://creativecommons.org/licenses/by/4.0/legalcode), which permits unrestricted, noncommercial use, distribution and reproduction in any medium, provided the work is properly cited. 